\title{
LA AGRICULTURA URBANA Y PERI-URBANA: UNA ALTERNATIVA PARA EL DESARROLLO LOCAL EN NICARAGUA
}

\section{Armando Flores García}

Ingeniero Agrónomo con orientación en Fitotecnia, Master en Ciencias Agrícolas con mención en Fitotecnia General. Master Internacional en Desarrollo Rural - Local

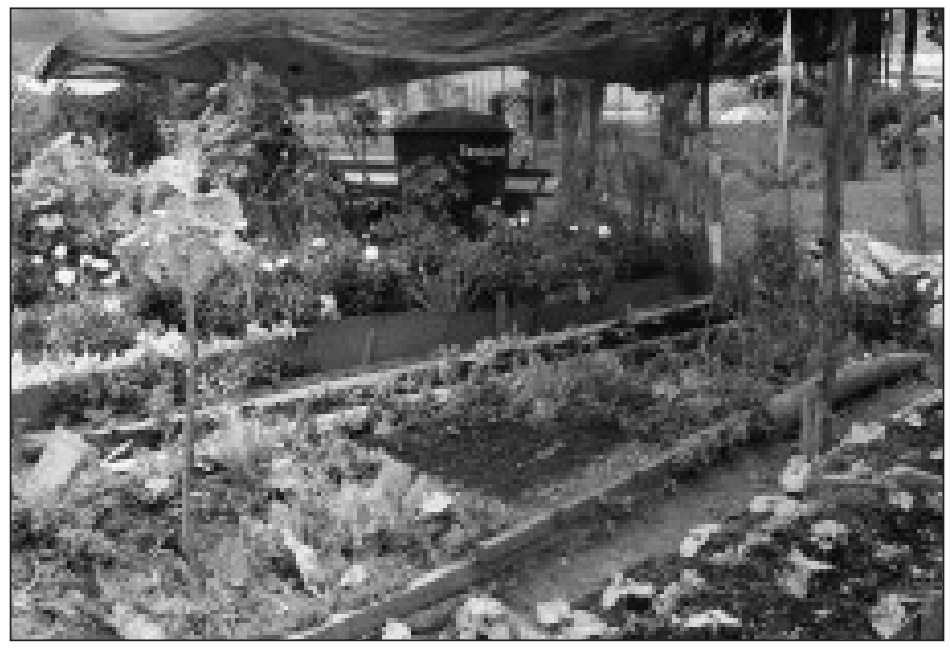

$\mathrm{S}$ i bien generalmente se considera que la agricultura es sólo una actividad rural, también suele ser un componente del sustento en las ciudades, que puede actuar como fuente de alimentación y empleo para familias pobres o incluso para toda una ciudad. Incluso en las áreas más hacinadas, las familias pueden a veces encontrar espacio para cultivar vegetales o criar animales y complementar así los alimentos que compra.

Lo que se presenta a continuación son algunas de las referencias contenidas en el Trabajo de Investigación " $\mathrm{La}$ Agricultura Urbana y Peri-Urbana como herramienta para la Seguridad Alimentaria y el Desarrollo Local en zonas Peri-Urbanas a la ciudad de Managua (Nicaragua)", presentado por el autor para la obtención del Título de Master Internacional en Desarrollo Rural - Local por la Universidad Politécnica de Madrid y la Fundación INFODAL (Instituto Internacional para la Formación y Desarrollo de los Agentes Locales), en Madrid (España), gracias a una beca obtenida a través de la Fundación Carolina.

La agricultura urbana y peri-urbana. De acuerdo con la FAO (citada por Méndez, Ramírez y Alzate, 2005), superficies (por ejemplo, solares, huertos, márgenes, terrazas, recipientes) situadas dentro de una ciudad y destinadas a la producción de cultivos y la cría de ganado menor o vacas lecheras para el consumo propio o para la venta en mercados de la vecindad".

Por su parte, la expresión Agricultura Peri-urbana se refiere a:"Unidades agrícolas cercanas a una ciudad que explotan intensivamente granjas comerciales o semicomerciales para cultivar hortalizas y otros productos hortícolas, criar pollos y otros animales y producir leche y huevos".

Al respecto, se ha demostrado que hasta un $50 \%$ de la población en las ciudades latinoamericanas tienen alguna participación en actividades vinculadas a la agricultura urbana o periurbana (IFPRI, 2004).

De acuerdo con la FAO (citada por Méndez, Ramírez y Alzate, 2005), lo que en algunos casos diferencia la agricultura urbana de la rural es su proximidad a grandes asentamientos humanos, circunstancia que crea a la vez oportunidades y riesgos. Según esta misma institución, entre las oportunidades se incluyen las siguientes: acceso a los mercados de consumo; menor necesidad de envasar, almacenar y transportar los alimentos; posibles empleos e ingresos agrícolas; acceso de los consumidores pobres 
a los alimentos por medios distintos del mercado; disponibilidad de alimentos frescos y perecederos; proximidad a los servicios, incluidas las instalaciones de tratamiento de desechos; y posibilidades de recuperación y reutilización de desechos.

Por su parte, entre los riesgos pueden anotarse los siguientes: riesgos para el medio ambiente y la salud derivados de prácticas agrícolas y acuícolas inadecuadas; aumento de la competencia por la tierra, el agua, la energía y la mano de obra; y reducción de la capacidad del medio ambiente para absorber la contaminación.

No obstante, es preciso destacar el papel de las Agriculturas Urbana y Periurbana (AUP ) en el tejido social de las comunidades donde se desarrolla. Así, por ejemplo, se ha enfatizado el papel de "la AUP como una actividad que produce alimentos para enfrentar el crecimiento del hambre, produce plantas medicinales facilitando el acceso a la salud, genera empleo de bajo costo, reduce la exclusión social valorizando la identidad individual y comunitaria” (Merzthal, 2004).

Además, con la $A U P$ se puede "Promover el comercio justo y solidario de productos limpios que mejore los ingresos de los pequeños productores", a la vez que se mejora la calidad higiénico-sanitaria y nutritiva de los alimentos consumidos por la población local (Vásquez, 2003).

Particularmente, "la actividad agrícola en las zonas periurbanas detiene la proliferación de asentamientos irregulares, crea una barrera verde y productiva, mejora el aire, la filtración de agua a los acuíferos locales, regenera espacios de recreo, dibuja por tanto, una ciudad con posibilidades de futuro" (Canabal, 2004).

Desde el punto de vista del impacto social de la $A U P$, se han definido algunas líneas básicas al respecto “da empleo a los ancianos jubilados y a personas incapacitadas de conseguir empleo debido a problemas mentales”, y puede brindar capacitación pre-profesional a estudiantes universitarios (Vásquez, 2004).

Adicionalmente, la AUP puede orientarse a la alimentación de cuerpos uniformados, como documenta Fuster (2004). Pero, también son conocidas las experiencias desarrolladas de integración al trabajo en AUP para involucrar a grupos vulnerables como jóvenes, mujeres, discapacitados, nuevos inmigrantes al área urbana, personas de la tercera edad y desempleados, con el objetivo de integrarlos más firmemente en el sistema urbano y proveerles así de un medio decente para ganarse la vida (ETC-RUAF, 2003).

La agricultura urbana como eje aglutinante en el desarrollo local. Méndez, Ramírez y Alzate (2005) han expresado que el fenómeno de la agricultura urbana, desde sus distintos ángulos, quiebra la exclusiva asociación ente agricultura y ruralidad, abriendo a su vez la posibilidad de integrar la actividad agrícola al propio quehacer urbano, generalmente caracterizado por el uso improductivo del suelo y la predominancia de un género de vida de tipo industrial-transformador.

Existen numerosas experiencias en las cuales la AUP ha funcionado como agente de cohesión y dinamización territorial.

En Rosario (Argentina), se observó como "la Huerta posibilitó, además, la generación de otros emprendimientos asociados, como la construcción de herramientas artesanales, hornos urbanos y postes de cemento", así como la puesta en funcionamiento de las Agroindustrias Urbanas Sociales, para darle valor agregado a la producción primaria, mejorando el ingreso de los productores urbanos, esta iniciativa sirvió posteriormente como Eje Aglutinante, por medio de la cual se sumaron a la experiencia de las autoridades locales, organizaciones de la sociedad civil, autoridades gubernamentales de diferentes niveles, centros de estudio e investigación, e incluso organismos internacionales y multilaterales (Municipalidad de Rosario, 2004)

En el caso de la experiencia de AUP en Cuenca (Ecuador), “(...) algunas de sus actividades son financiadas a través del presupuesto participativo y congrega iniciativas de las comunidades, de la sociedad civil y el sector empresarial, promoviendo procesos participativos en la implementación de las políticas locales democráticas y equitativas. Actualmente, el programa viene reforzando la organización comunitaria, brinda capacitación y asistencia técnica. Asimismo, cuenta con una línea de micro crédito para los pequeños productores y apoya la comercialización con el mejoramiento de mercados y ferias libres y la Red de Economía Solidaria” (Vásquez, 2004).

Dentro de un sistema político y de organización socioeconómico diferente, "La producción de AU en Cuba tiene un sistema de aseguramiento técnico material que permite lograr una sostenibilidad en la producción contando con consultorías, clínicas, producción de materia orgánica, centro de producción de medios biológicos, áreas colectivas de las cooperativas, casas de postura (aseguramiento al productor), centro de promoción a la AU (...), aulas de capacitación (15 aulas en la ciudad) y 252 extensionistas en los consejos populares" (Fuster, 2004), lo cual, no obstante, refleja la amplia variedad de emprendimientos que pueden surgir alrededor de las iniciativas de AUP . Algunos ejemplos 
exitosos de empresas de este tipo, en ciudades tan disímiles como Cuenca (Ecuador) y Dar-es Salaam (Tanzania) pueden apreciarse en el Video sobre Agricultura Urbana de la ETC-RUAF (2003) y otros documentos de esa misma fuente, incluyendo las ediciones sucesivas de la Revista de Agricultura Urbana.

Otro aspecto fundamental de los efectos de la AUP en el tejido social de los territorios, es destacado por Canabal (2004): "Identidades que se reconstruyen con los nuevos contactos culturales -urbanos y de otras partes del país-de los que han aprendido la manera de adaptar a las nuevas condiciones sus espacios, su aprovechamiento, el tipo de productos, su misma capacitación, la posibilidad de organizarse y de tejer redes con otras organizaciones, con agentes de gobierno $y$ con agencias no gubernamentales que les ofrecen acompañamiento y una vinculación necesaria. Estos actores, que se han ido conformando en las fronteras de lo urbano con lo rural, pertenecen al mundo de hoy en que combinan sus actividades agropecuarias con su vida en la ciudad, donde la familia se emplea en una gran diversidad de empleos; combinan lo que fueron, su historia, su herencia tecnológica, con las posibilidades que les ofrece la ciudad: urbanos, rurales, rural urbanos; quizás en esta combinación, dadas las condiciones del empleo estrictamente urbano, encuentren las posibilidades de su futuro."

La agricultura urbana en Managua. Tradicionalmente, en el área urbana de la Ciudad de Managua se han desarrollado diversas iniciativas particulares de producción agropecuaria. En algunos casos, debido a que el desarrollo de la ciudad misma ha terminado "urbanizando" pequeñas propiedades agropecuarias, cuyos propietarios, no sin razón, se resisten a abandonar su forma de vida tradicional. En otros casos, los pobladores inmigrantes de áreas rurales de alguna manera desean, de manera consciente o inconsciente, mantener algún vínculo con su forma de vida anterior. Aún hay otros, quizás la mayoría de los pobladores urbanos, que sienten la necesidad de "humanizar" la ciudad o de complementar la dieta familiar, ya sea con la crianza de animales o con el cultivo de frutas, hortalizas, y plantas medicinales o aromáticas.

Durante inicios de la década de los años 80, durante la “Revolución Popular Sandinista”, el Programa Mundial de Alimentos (PMA) del Sistema de Naciones Unidas, en coordinación con el entonces Ministerio de Desarrollo Agropecuario y Reforma Agraria (MIDINRA), desarrolló el Programa Alimentario Nacional (PAN), que entre otras actividades de promoción a la diversificación de la dieta familiar, cultivó un "Huerto Popular" en una céntrica área de la Ciudad de Managua, conocida entonces como la "Plaza 19 de Julio", además que impulsó el establecimiento de los "patios urbanos y peri-urbanos", así como algunos "huertos comunales".

Ante cambios en las políticas de los diferentes organismos participantes en el PAN, a mediados de los años 80, el Huerto de la Plaza 19 de Julio pasó a manos del entonces Ejército Popular Sandinista (EPS), quien lo asimiló dentro del "Proyecto Agropecuario de la III Región Militar". La idea del ejército con este proyecto era producir alimentos básicos (legumbres y vegetales) de alta calidad e integridad para las unidades militares de la región, sus tropas y familias, generando además un importante flujo de los mismos productos a los mercados capitalinos. El proyecto agropecuario contaba con otras cuatro unidades de producción en el área peri-urbana de Managua, además de un Proyecto de Extensión del Sistema de Producción "de patios urbanos" en cinco barrios de la ciudad (información personal).

Luego de las elecciones de 1990 y a consecuencia de los cambios políticos, económicos y sociales resultantes de ellas, el Huerto Regional del antiguo EPS (convertido ahora en Ejército de Nicaragua) fue desmembrado $\mathrm{y}$ algunas de sus tierras fueron transformadas en un proyecto habitacional, otras vendidas a particulares, o adjudicadas a las universidades Centroamericana (UCA) y Nacional de Ingeniería (UNI), así como a la Escuela Nacional de Danza, e incluso otras fueron objeto de litigios de propiedad (Mairena, 2001), perdiéndose con ello esta interesante experiencia de AUP.

Posteriormente, algunos ONG's fomentaron la práctica de los huertos comunales en algunos barrios con poblaciones altamente vulnerables en diversas zonas de la ciudad de Managua, haciendo uso fundamentalmente de la técnica de “Cultivos Hidropónicos”. Sin embargo, esa práctica duró mientras fluyeron recursos de los organismos de cooperación externa.

A partir de los últimos años se ha retomado tímidamente la idea, destacándose entre estas iniciativas la Granja Integral “Las Granjita” (CIPRES, 2005) del Centro para la Promoción, la Investigación y el Desarrollo Rural y Social (CIPRES), aunque ésta tiene más un fin demostrativo de las prácticas que ese organismo promueve en el sector rural, que en el sentido estricto de Agricultura Urbana.

En este sentido, se debe destacar además la experiencia actualmente en marcha de un Proyecto comunitario desarrollado por pobladores de una zona del Municipio de Ciudad Sandino.

Sistemas de producción basados en el cultivo de frutales y hortalizas y la crianza de animales menores en los predios de las casas, dentro y en la periferia de las ciudades. 
Así mismo, luego del paso del Huracán “Mitch” en Octubre de 1998, el Programa Campesino a Campesino (PCaC), de la Unión Nacional de Agricultores y Ganaderos (UNAG), desarrolló un "Programa de restablecimiento productivo del sistema de fincas periurbanas impactadas por el Huracán Mitch en Estelí, Madriz y Nueva Segovia” (Información personal).

Más recientemente, la FAO, a través del Programa Especial para la Seguridad Alimentaria (PESA), estableció un protocolo de colaboración con la Alcaldía de Managua para impulsar un proyecto conjunto de agricultura urbana y peri urbana con las familias más pobres en los barrios marginales, impulsando el patio productivo y una especial vinculación con la creación de huertos escolares en zonas marginales de la capital (FAO, 2005).
Reflexiones finales. Managua es una ciudad que se ha ido modernizando en cuanto a su infraestructura vial y comercial, pero donde subsisten agudos problemas sociales derivados del desempleo, la marginación y la inseguridad alimentaria de importantes sectores populares, además de crecientes problemas originados, entre otros factores, por el desordenado crecimiento de la ciudad, la destrucción del medio ambiente circundante y las limitaciones en las redes de servicios públicos.

La Agricultura Urbana y Peri-urbana, si se integra en los planes de Ordenamiento Territorial de las Municipalidades y es ejecutada con el apoyo a largo plazo de Instituciones y programas con un claro enfoque

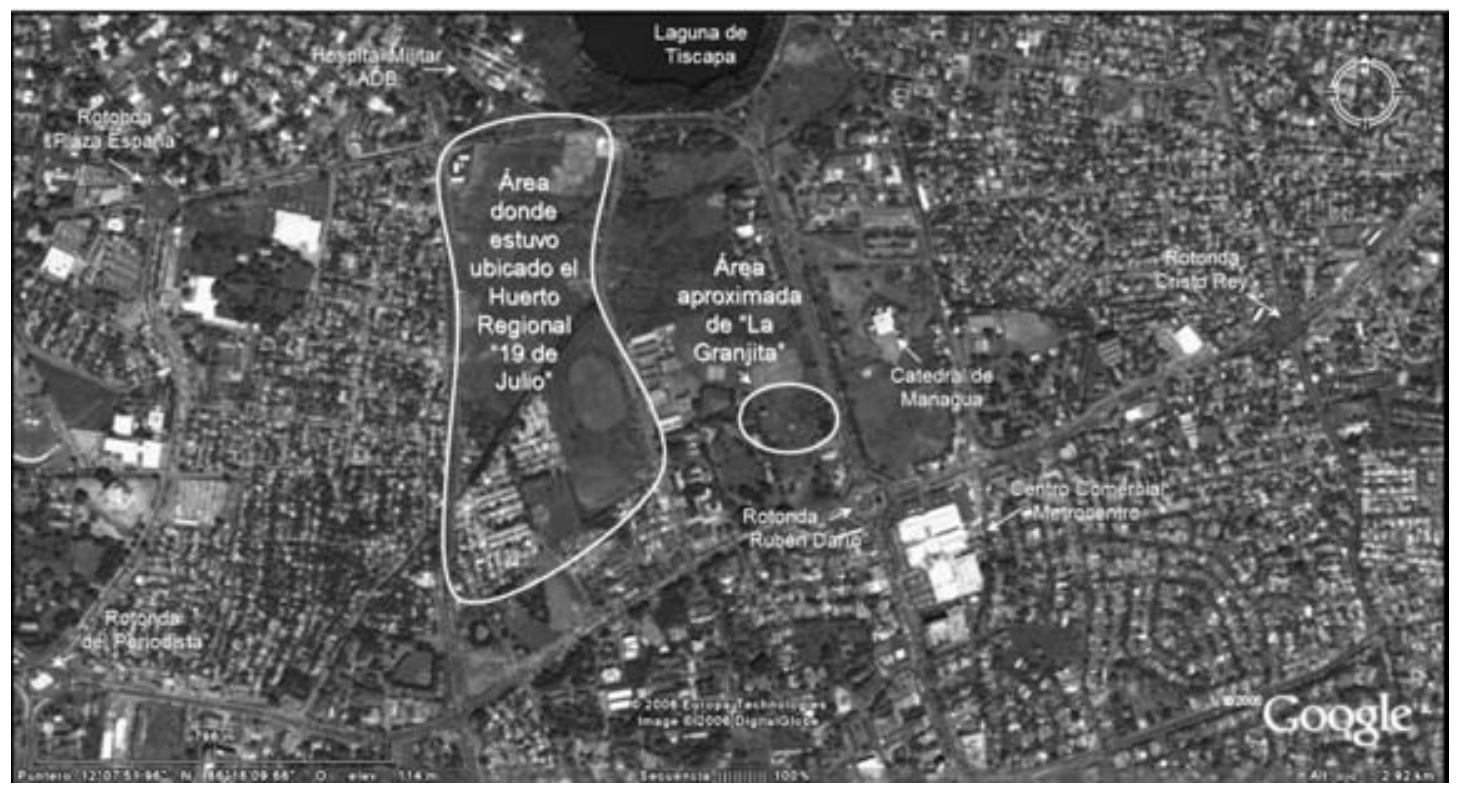

Figura 1. Imagen satelital del centro de la Ciudad de Managua, mostrando la ubicación aproximada donde estuvo el Huerto Regional "Plaza 19 de Julio" y donde se encuentra "La Granjita” del CIPRES. Fuentes: Programa Google Earth e Información Personal.

Así mismo, la Cámara Nicaragüense Americana (AMCHAM), el IICA y la FAO, están desarrollando un Programa de Huertos Escolares, para lo cual cuentan con el apoyo del Ministerio de Educación, Cultura y Deportes (MECD). La ejecución de este programa de huertos tiene como objetivo principal enseñar a producir la tierra a los estudiantes, profesores y padres de familia, aportando al mismo tiempo con alimentos para los niños y niñas de los centros escolares. En el año 2007, para comenzar el programa, el IICA donó semillas de hortalizas, fertilizantes y herramientas agrícolas a 12 de las 107 escuelas con las cuales se espera iniciar este año el establecimiento de huertos (IICA, 2007). territorial y visión de nación, podría dar como resultado la dinamización del capital humano y social del territorio, dando origen al embrión para un desarrollo local a mediano plazo. Simultáneamente y de manera inmediata, se beneficiaría más directamente a la población local vulnerable mejorando su seguridad alimentaria, con la provisión de alimentos frescos, sanos, y seguros, además de generar empleos, bienes y servicios, a bajos costos y siempre en el propio medio local.

En particular y considerando la actual coyuntura del país, sería provechoso aprovechar las oportunidades que brindan los convenios de cooperación firmados con los países miembros del ALBA (Alternativa Bolivariana 
para las Américas), particularmente Cuba, Venezuela y Ecuador, quienes tienen experiencias amplias y documentadas en cuanto al desarrollo de Agricultura Urbana y Peri-Urbana, sin menospreciar los aprendizajes de nuestro entorno que puedan aún sistematizarse.

Así mismo, sería necesario fortalecer los proyectos que actualmente se estén ejecutando en este sentido, en particular los que han nacido de propia iniciativa de las comunidades, sistematizando además los aprendizajes que de ellas se obtengan, y simultáneamente coordinar los convenios firmados entre instituciones nacionales con organismos internacionales y multilaterales, para que todas estas acciones resulten exitosas y sostenibles en el largo plazo.

Finalmente, considero que Nicaragua tiene todo el potencial favorable para iniciar el camino hacia un Desarrollo Local diverso, endógeno y sostenible, sólo es necesario que se le entreguen las herramientas precisas a sus comunidades, y la Agricultura Urbana y Peri-urbana parece ser una herramienta apropiada para Managua y sus poblaciones aledañas.

\section{REFERENCIAS BIBLIOGRÁFICAS}

Méndez, Marlon; Ramirez, Luz; Alzate, Alejandra. 2005. La Práctica de la Agricultura Urbana como expresión de emergencia de nuevas ruralidades: reflexiones en torno a la evidencia empírica. Cuadernos de Desarrollo Rural, №55, Bogotá (Colombia), Segundo semestre, p.51-70.

IFPRI (Instituto Internacional para la Investigación sobre Políticas Alimentarias). 2004. Vida Urbana: Retos y Opciones para los Pobres en la Ciudad. Issue Brief: 9, Washington, D. C. (USA).

Merzthal, Gunther. 2004. La Agricultura urbana (AU): motor para el desarrollo municipal sostenible. En: COSECHA URBANA. Memoria y Declaración: “La Integración de la Agricultura Urbana en el Desarrollo Sostenible de las Municipalidades”. Lima, Perú, 26 de Noviembre del 2003. Centro Internacional de la Papa, Lima (Perú), p. 3.

Vásquez, María Caridad. 2004. Experiencia de AU en Cuenca-Ecuador. En: COSECHA Urbana. Memoria y Declaración: “La Integración de la Agricultura Urbana en el Desarrollo Sostenible de las Municipalidades”. Lima, Perú, 26 de Noviembre del 2003. Centro Internacional de la Papa, Lima (Perú), p. 3-4.

Canabal C., Beatriz. 2004. La Agricultura Urbana Hoy. Una Reflexión desde Azcapotzalco. En: ARIAS H., Gabriela; CANABAl C.; Beatriz; BARRERA G., Rafael; MUÑoZ A., Octavio; HERNÁNDEZ M., Oralia; ARROYO G. D., Francisco J.; SORIANO R., RAmÓn (Eds.). Agricultura Urbana y Periurbana en México. Segundo Encuentro Nacional. La Agricultura Urbana como Estrategia Organizativa para el Abasto de Alimentos, la Supervivencia Familiar-Local, la Sostenibilidad Ambiental y las Políticas Públicas. Universidad Autónoma de Chapingo, Chapingo (México), p. 9-14.

Fuster, Eugenio. 2004. Agricultura Urbana en Cuba. En: COSECHA Urbana. Memoria y Declaración: "La Integración de la Agricultura Urbana en el Desarrollo Sostenible de las Municipalidades”. Lima, Perú, 26 de Noviembre del 2003. Centro Internacional de la Papa, Lima (Perú), p. 4-5.

ETC-RUAF (Centro de Recursos en Agricultura y Forestería Urbanos). 2003. Vídeo sobre Agricultura Urbana. World Report Foundation, Ámsterdam (Holanda). CD-ROM.

Municipalidad de Rosario; Centro de Estudios de Producciones Agroecológicas. 2004. Rosario Cultiva. Programa de Agricultura Urbana. Rosario (Argentina), 18 minutos.

Mairena Martínez, Mario. 2001. Surge “dueño” y cerca la "Plaza 19 de Julio”. El Nuevo Diario, Nacionales, Managua (Nicaragua), 31 de mayo, 2001 http://archivo.elnuevodiario.com.ni/2001/mayo/31-mayo-2001/nacional/nacional2. html

CIPRES (Centro para la Promoción, la Investigación y el Desarrollo Rural y Social). 2005. Granja Integral del CIPRES - “La Granjita”. Managua (Nicaragua). http://www.cipres.org/

FAO (Organización de las Naciones Unidas para la Agricultura y la Alimentación). 2005. Ayudando a construir un mundo sin hambre. Informe de Gestión 1980-2005 de la FAO en Nicaragua. Managua (Nicaragua).

IICA (Instituto Interamericano de Cooperación para la Agricultura). 2007. Huertos Escolares Prioritarios para el MECD. IICA Nicaragua Agronoticias (Boletín Electrónico), Managua (Nicaragua), \# 193, Semana del 26 de enero al 2 de febrero. 